DOI: $10.14746 /$ por. 2018.1 .8

\title{
VIELSTIMMIGE JÜDISCHE ERZÄHLUNGEN IN DER ZEITGENÖSSISCHEN POLNISCHEN LITERATUR
}

\author{
Hans-Christian Trepte, Iris Tabea Bauer ${ }^{1}$ \\ (Universität Leipzig)
}

\begin{abstract}
Keywords: Holocaust, Jews, history, literature, culture, identity, fiction, narrative Słowa kluczowe: Zagłada Żydów, holokaust, historia, literatura, kultura, tożsamość, analiza, fikcja, narracja
\end{abstract}

\begin{abstract}
Hans-Christian Trepte, Iris Bauer, VIELSTIMMIGE JÜDISCHE ERZÄHLUNGEN IN DER ZEITGENÖSSISCHEN POLNISCHEN LITERATUR. "PORÓWNANIA" 1 (22), 2018. Vol. XXII, P. 145-168. ISSN 1733-165X. The Representation of the Holocaust in Polish literature can be pursued from World War II up to contemporary narratives. The authors of this text concentrate on important debates in Poland concerning first of all the common as well as separate Polish-Jewish past in history, but mainly in times of the German fascist occupation. Following their literary studies they give a brief survey of the mentioned topic, concentrating mainly on three exemplary Polish books: Joanna Bator Chmurdalia (2010), Jarosław Kamiński Rozwiazła (2011), and Igor Ostachowicz Noc żywych Żydów (2012). The literary analysis of these texts is related to Polish history, culture, and politics, concerning first of all crucial changes in the society and culture. The chosen writers and their works demonstrate different approaches to an uncertain, complicated Polish Jewish past. They illustrate important changes in literature towards documentary and fictional narratives. More and more tabooed topics of the extreme living conditions during the Nazi occupation in and outside of the concentration camps are considered - crime, various forms of sexuality and prostitution. Jewish life stories as well as the holocaust turn meanwhile into ,normal ${ }^{\prime}$ motives in contemporary Polish narratives. Searching for their own roots, identity and family traces on the basis of documents, reports or photos often results in colourful reconstructions of Polish-Jewish fates, a striking tendency not only in contemporary Polish literature.
\end{abstract}

1 E-mail: treptus@t-online.de, iris.bauer@geschichte.uni-halle.de 
Abstrakt: Hans-Christian Trepte, Iris Bauer, VIELSTIMMIGE JÜDISCHE ERZÄHLUNGEN IN DER ZEITGENÖSSISCHEN POLNISCHEN LITERATUR. „PORÓWNANIA” 1 (22), 2018. T. XXII, S. 145-168. ISSN 1733-165X. Zagłada Żydów zajmuje bardzo ważne miejsce w polskiej literaturze powojennej od czasów drugiej wojny światowej aż do współczesności. Autorzy artykułu koncentrują się przede wszystkim na ważnych debatach dotyczących polsko-żydowskiej przeszłości, zwłaszcza czasów okupacji faszystowskiej. W centrum zainteresowania znajdują się wybrane teksty współczesnej literatury polskiej analizowane w szerokim kontekście historycznym, społeczno-politycznym i kulturowym. Rozważono, w jaki sposób przedstawiciele różnych pokoleń podchodzą do tematyki Żydów w Polsce oraz Zagłady. W analizach skoncentrowano się na trzech znaczących dziełach literackich: Chmurdaliach Joanny Bator (2010), Rozwiązłej Jarosława Kamińskiego (2011) i Nocy żywych Żydów Igora Ostachowicza (2012), które prezentują różne podejścia pisarzy do skomplikowanej polsko-żydowskiej przeszłości oraz ilustrują istotne przesunięcia w sposobie jej opisywania. Zauważono zmianę w podejściu do tabuizowanych tematów warunków życiowych w obozach koncentracyjnych (przestępstwa, przemoc seksualna, prostytucja) oraz traktowaniu żydowskości, która staje się ważnym, lecz nie dominującym motywem literackim. W najnowszej literaturze poszukiwanie korzeni oraz tożsamości żydowskiej na podstawie dokumentów czy zdjęć często skutkuje barwnymi rekonstrukcjami polsko-żydowskich losów.

\section{Zum ,jüdischen’ Erzählen nach 1989}

Mit dem Zusammenbruch des kommunistischen Regimes setzen in Polen wie auch in anderen Staaten des Ostblocks ab 1989/90 nicht nur politische, gesellschaftliche und wirtschaftliche Transformationen ein, sondern es beginnt auch eine grundlegende Revision, eine deutliche Befreiung von der kommunistischen Geschichtsdoktrin. In Bezug auf den Holocaust und die Geschichte der Jüdinnen und Juden in Polen geht es dabei unter anderem auch um die Loslösung der jüdischen Leidensgeschichte aus einer vor allem abstrakt dargestellten angeblichen polnisch-jüdischen Opfergemeinschaft. Die einzelnen Schicksale und der massenhafte Tod polnischer Jüdinnen und Juden wurden von der offiziell propagierten Erinnerungspolitik in der Volksrepublik Polen oft bis zur Unkenntlichkeit in polnische Opfer-Narrative integriert und damit sozusagen einseitig polonisiert.

Das tabuisierte, schlicht verbannte Wahrnehmen, Aus-/Ansprechen und Anerkennen einer eigenständigen jüdischen ,Geschichte' und ,Identität', nicht nur auf den Holocaust bezogen, entlud sich nach dem demokratischen Umbruch in Polen im gesellschaftlichen, kulturellen und literarischen Diskurs somit mit einer Verspätung von fast 50 Jahren und erklärt, warum der polnische Literaturwissenschaftler Przemysław Czapliński auch noch 2010 zurecht äußert: „II wojna światowa trwa nadal. My ją toczymy, a ona toczy nas" (Czapliński 2010: 337). Die Auseinandersetzung mit polnischen Besatzungs- und Kriegstraumata ist nicht nur von der Last geprägt, zum Epizentrum des Krieges und des Holocaust gemacht und zur Augenzeugenschaft gezwungen worden zu sein, nationalsozialistische und sowjetische Okkupationserfahrung verarbeiten $\mathrm{zu}$ müssen, sondern wird auch durch die er- 
zwungene Verzögerung der Aufarbeitung und die manipulative Modellierung der Geschichtsschreibung durch das kommunistische Regime erschwert. Nach 1989 beginnt folglich ein Prozess, der ähnlich einer Spurensuche nach einer neuen, anderen Wahrheit fragt, bzw. unter dem Teppich des kommunistischen Mega-Narrativs des Zweiten Weltkriegs und des Holocaust andere, verdrängte und marginalisierte Geschichten bzw. Narrative hervorzuholen versucht. In der Literatur spiegelt sich dieser Prozess in einer Welle von späten Zeugnissen von Holocaust-Überlebenden wider, die sich auch durch die stärkere Verlagerung weg vom Kollektivdenken hin zu individuellen Schicksalen und Erfahrungen, aber auch durch vermehrtes Schreiben von Frauen von vorherigen Zeugnisaussagen unterscheiden. Außerdem wird der polnische Inlands-Diskurs, auch unter Berücksichtigung der zweiten Zirkulationsebene polnischer Literatur (drugi obieg) durch die im nun ehemaligen Exil entstandene und jetzt auch im Lande selbst frei zugängliche Literatur nicht nur polnischer Provenienz verstärkt. Der Völkermord an den polnischen und europäischen Jüdinnen und Juden ist dabei zu einem scheinbar unerschöpflichen Inspirationsquell, einem "globalen Referenzpunkt" (Probst 45) geworden.

Die in den Werken von Hanna Krall beginnende Tendenz Lebensbeichten, authentische Berichte und Geschichten von Holocaust-Überlebenden aufzuzeichnen, die selbst nicht in der Lage waren, dies zu tun, wird zu einem tragenden, zentralen Motiv in der polnischen zeitgenössischen Literatur über den Holocaust und zur jüdisch-polnischen Thematik generell. Das Nacherzählen aus zweiter Hand wird in der jüngeren polnischen Literatur über den Holocaust weiter fortgesetzt, Authentisches und Nacherzähltes, Facta und Fikta vermischen sich. Und immer häufiger wird sogar auf eine authentische Zeugenschaft verzichtet bzw. kann diese bewusst erdacht werden. Eine Tendenz, die bei polnischen Vertreter_innen der mittleren und jüngeren Schriftstellergeneration besonders deutlich zu beobachten ist.

DieseEntwicklung innerhalbPolens kann mit den Entwicklungen der ,Holocaust-Literatur' im Allgemeinen in Einklang gebracht werden. In den 1990er Jahren wird nicht nur die ,Geschichte' des Holocaust zunehmend umgeschrieben, ist er doch bereits zu einem "Mythos der Moderne“ (Assmann, Frevert 272) geworden, sondern es verändert sich $u$. a. auch das Opfer-Täter-Verhältnis. Im internationalen Diskurs spielt diesbezüglich vor allem Jonathan Littells Buch Les Bienveillantes (2006) eine besondere Rolle. Saul Friedländer ordnete das Werk in eine Typologie des stetig zunehmenden kommerzialisierten Holocaust-Kitsches ein (Szczęsna 4). In diesem Zusammenhang muss auch eine besondere Form der Kommerzialisierung, der absoluten Vermarktung des Holocaust in Gestalt einer ausgeprägten „Holocaustindustrie“ (Finkelstein) erwähnt werden. Denn mit Prozessen der Enttabuisierung, der absoluten Fiktionalisierung und Vermarktung, geht auch die Gefahr einher, dass der Holocaust-Diskurs an Wahrheit, Ernst und Authentizität verliert: „In the light of media over-exposure the evil oft he Holocaust becomes strangely weight- 
less“ (Hartmann 11). Hinzu kommt die Thematisierung der „Holocaustprofiteure“ (Bajohr/Löw) und des „Holocaustkitsches“ (Clendinnen 20) sowie die sich häufende Verwendung des Holocaust als „decorum“ (Engelking 79).

Insgesamt wächst in der Literatur der neunziger Jahre jedoch vor allem das Bedürfnis nach „Personalisierung, nach Privatisierung und Intimisierung" und es wächst die Tendenz hin zum „Politainment“ (Dörner 1-2), welche mit einer spürbaren Sehnsucht nach „Entlastung", „Vergebung" und „Erlösung" einhergeht (Reichel 315). Etwa seit Mitte der neunziger Jahre „begannen sich die Vorstellungen bzw. die als verbindlich angesehenen Muster, nach denen über den Holocaust zu schreiben sei, zugunsten einer neuartigen Polyphonie aufzulösen" (Trepte 2015: 144), wodurch eine Vielfalt von sehr unterschiedlichen literarischen Werken ensteht.

Im Kontext dieser Entwicklungstendenzen und der ,politischen' Öffnung des polnisch-jüdischen Diskurses, wird für die jüngste Vergangenheit Jan Tomasz Gross' Publikation Sasiedzi aus dem Jahr 2000 von der Forschung als die entscheidende, wichtige Zäsur des neuen Millenniums beschrieben, da die von ihm ausgelöste Diskussion „[...] przekroczyła [ona] temperaturę poprzedniej ważnej debaty o polskożydowskiej przeszłości okupacyjnej, sprowokowanej artykułem Jana Błońskiego Biedni Polacy patrza na getto" (Kowalska-Leder 768). Nach Błońskis entscheidendem und bahnbrechendem Essay erreichte Jan Tomasz Gross mit seinem Buch über die Verbrechen in Jedwabne eine neue Stufe im polnischen Diskurs², die ebenso großen Einfluss auf die polnischsprachige Literatur über den Holocaust zeigen sollte.

Die von Gross angestoßene Öffnung und Enttabuisierung des Diskurses bedeutet für die polnische ,Holocaust-Literatur' eine immer freiere Verarbeitung des Themas, wobei auch die größer werdende zeitliche Distanz und die fortschreitende Entwicklung der historiographischen Aufarbeitung eine nicht zu unterschätzende Rolle spielen. Das Zusammenspiel dieser Faktoren führt $u$. a. dazu, dass immer weniger die faktische Geschichte, sondern das heutige Gedächtnis, die geschaffene Erinnerung, im Zentrum der Literatur über den Holocaust steht, was sich auch im Schreiben der Kinder- und Enkelgeneration der Holocaustüberlebenden beobachten lässt. Die biographische Spurensuche nach der eigenen Herkunft wird durch die freiere Auseinandersetzung mit der Gegenwart und der persönlichen Verarbeitung dieser ergänzt. So nimmt etwa seit der Jahrtausendwende die Zahl der publizierten Erinnerungen zwangsläufig ab. Stattdessen kommt es zur „Publikation von fiktionalen Bearbeitungen durch gänzlich Unbetroffene aus der zweiten, dritten oder auch vierten Generation nach der Erlebnisgeneration“ (Roth 19). Es kommt „zu einem

2 Eine Auseinandersetzung mit der polnischen Mitschuld und Augenzeugenschaft fand schon während des Krieges, im Untergrund und im Exil statt, in der Volksrepublik Polen jedoch im offizielleren Rahmen erst ab den 1980er Jahren. Zeugnis der im öffentlichen Diskurs aufkommenden Hinwendung zur jüdischen Geschichte ist neben Jan Blońskis Essay Biedni Polacy patrza na getto auch die TV-Ausstrahlung Claude Lanzmanns Film Shoah im selben Jahr. 
spürbaren Adressaten- und Paradigmenwechsel [...] von authentischen Zeugenaussagen hin zur sekundären Zeugenschaft der Kinder und Enkel der Holocaustüberlebenden" (Trepte 2015: 143).

Dabei spielt in der Auslands- wie auch in der polnischen Inlandsliteratur Intermedialität - vor allem in Gestalt von den Texten beigefügten Dokumenten, Bildern, Fotos, Karten u. a. als Beweisstücke bzw. authentische Zeugnisse - eine wichtige Rolle im Erzählen über jüdische Schicksale und den Holocaust. Verwiesen sei in diesem Kontext beispielsweise auf Anda Rottenbergs Autobiographie Proszę bardzo (2009) sowie auf den mit dem Astrid-Lindgren-Manuskriptpreis und von der Internationalen Jugendbibliothek in den White Ravens Katalog aufgenommenen (Kinder) Roman Arka czasu (2013) von Marcin Szczygielski. Das Buch verbindet aus der Erzählperspektive des achtjährigen Rafał, der mit seinem Großvater im Warschauer Ghetto lebt, Fantasy und Holocaust miteinander. Die Intermedialität der ,HolocaustLiteratur' bedeutet auch ihre Enttabuisierung: Es ist nicht nur eine größere inhaltliche Bandbreite an dem, was gesagt werden darf, erlaubt, sondern auch eine Freiheit in der Konzeption eben dieser Literatur. Neue Herangehensweisen (in Form und Inhalt) - in Krimi, Film, Komödie, Operette, graphic novels, Comics u. v. m. - treiben die Fiktionalisierung der Narrative über den Holocaust bis ins Absurde, Mystische oder Groteske und tasten damit auch die moralischen Grenzen des Genres der ,Holocaust-Literatur' neu ab (Trepte 2015: 143).

So nimmt sich nunmehr auch die Populärkultur in einem immer stärker werdenden Maße der Thematik des Holocausts an. „,Unangemessene' Gattungen und Kategorien", und dazu zählte anfangs auch der Krimi, werden zunehmend in den „Bereich der Holocaust-Kunst" eingeführt (Głowiński 8). Als Beispiel sei die erfolgreiche Krimitrilogie Zygmunt Miłoszewskis (Ziarno prawdy, Uwikłanie und Gniew) genannt: Eine kritische, reflektierte Darstellung weit verbreiteter antisemitischer Ressentiments, die sich unter dem Deckmantel von katholischer Kirche und engstirnigem, konservativem polnischen Nationalismus erneut ausbreiten können. Die Diskussion über die Holocaustthematik im polnischen Krimi steht dabei im Kontext der Debatte um die zunehmende mediale Transformation des Holocaust, die bereits seit dem Ende der siebziger bzw. zu Beginn der achtziger Jahre vor allem im Zusammenhang mit Fernsehserien wie Holocaust (1974) und der Erstausstrahlung von Claude Lanzmanns Shoah (1985) diskutiert wurde ${ }^{3}$. In Polen entstand vor allem in den letzten Jahren eine Reihe stark polarisierender Filme wie z. B. Jan Kidawa-Błońskis Różyczka (2010), Pasikowskis bereits erwähnter Pokłosie (2012), Wojciech Smarzowskis Róża (2012) und Paweł Pawlikowskis Ida (2013).

3 „After a long period of marginalization and general silence of Polish discourse, something changed. We witnessed the double overcoming of Jewish absence - absence caused by annihilation on the one hand, and absence caused by censorship an exclusion from public communication oh the other hand“ (Krupa 2015: 32). 
Eine weitere und für diesen Aufsatz zentrale Enttabuisierung der zeitgenössischen polnischen Literatur über den Holocaust bezieht sich auf die Identität, die Herkunft der Protagonist_innen. Zumeist entdecken diese, oft völlig unerwartet, ihre eigentliche jüdische Identität. Sie beginnen nach ihren wahren Eltern und Verwandten (und damit auch nach ihrem eigenen Ich) zu suchen. Oft sind diese literarischen Werke in einem gesamtpolnischen bzw. internationalen Kontext angesiedelt, d. h. die jüdische Thematik wie auch der Holocaust bilden zwar zentrale Motive, die sich allerdings in ein ganzes Geflecht aus verschiedenartigen anderen Themen und Motiven einordnen. Ein Beispiel hierfür ist u. a. Marian Pankowski, der erst in seinem Spätschaffen auf das jüdische Thema zurückkommt. In der 2007 in Twórczość erschienen Erzählung Nie ma Żydowki, die seiner Frau gewidmet und an deren Holocausterinnerung angelehnt ist, setzt sich Pankowski als nicht-jüdischer Überlebender mehrerer Konzentrationslager mit der Vermittlungs- und Kommunikationsproblematik der Holocausterfahrungen auseinander.

Aus dem bisher dargestellten Einblick in die komplexen Entwicklungstendenzen der zeitgenössischen polnischen Literatur über den Holocaust sollen nun exemplarisch drei Texte herausgegriffen werden, in denen das enttabuisierte fiktionale Schreiben als Potential verstanden wird, gemeinsame polnisch-jüdische Geschichten zu schreiben, Alteritäten immer mehr aufzulösen oder spielerisch zu verarbeiten. Die im Folgenden diskutierten Textbeispiele nicht-jüdischer, polnischer, im Jahr 1968 geborener Autor_innen (also knapp der dritten Generation nach dem Holocaust) - Joanna Bator, Jarosław Kamiński und Igor Ostachowicz - werden als Teil der enttabuisierten Literatur über den Holocaust interpretiert, in der der Holocaust sowie allgemein, ,Jüdischkeit', ,das Jüdische' in verschiedenen Textsorten und Medien, so auch dem Film, vermehrt als inhärenter und untrennbarer Teil ,des Polnischen' bzw. von ,Polonität' verhandelt, thematisiert, aufgearbeitet und verarbeitet wird. Bei der Wieder- bzw. Neuaneignung der Geschichte als einer gemeinsamen, kann verstärkt ein hierarchiebefreites mehrstimmiges Neben- und Ineinander verschiedener Erzählstränge beobachtet werden, wie es v. a. in Joanna Bators Chmurdalia der Fall ist. In dem 2010 in Polen und 2013 in Deutschland unter dem Titel Wolkenfern erschienenen Roman ist die jüdische Familiengeschichte der Protagonistinnen Dominika und ihrer Mutter Jadzia Chmura nicht nur in deren narrativen Identitätskonstruktionen ein Erzählstrang neben anderen. Auch in der Erzählstruktur des gesamten Textes tauchen jüdische Narrative neben $u$. a. postkolonialen und postkommunistischen auf, ohne eine spezifische Stellung zu behaupten. Bei Jarosław Kamińskis Rozwiazła (2011) besteht das enttabuisierte Schreiben darin, dass der kein Tabu scheuende Umgang mit Körperlichkeit und freier Sexualität mit dem Erzählen des Holocaust und der späten Entdeckung der eigenen jüdischen Identität kombiniert wird. Kamiński geht damit nicht nur Tabuthemen der polnischen ,HolocaustLiteratur' an, sondern wählt auch in Bezug auf den Umgang mit Körper, Sexualität und Kriminalität einen überraschend freizügigen Ton für die polnische Literatur im 
Allgemeinen. Als letztes Beispiel soll Igor Ostachowiczs Noc Żywych Żydów (2012) besprochen werden, welches als eines der spektakulärsten Beispiele für die Enttabuisierung des Holocaust stehen kann. In seinem ebenso wie Kamińskis Rozwiązła noch nicht ins Deutsche übersetzten Text steigen tote Jüdinnen und Juden aus den Kellern des auf dem Gebiet des ehemaligen Ghettos nach dem Krieg hochgezogenen sozialistischen Wohnblöcken im Warschauer Viertel Muranów wie aus dem verdrängten Unbewussten der polnischen Gesellschaft. Dabei wird über eine Mischung aus Horror-Fiction und Pop nicht nur äußerst kritisch Bezug genommen auf den Umgang Polens nach 1945 mit seiner jüdischen Vergangenheit und die Schicksale der polnischen jüdischen Bevölkerung während des Holocaust, sondern es wird auch die jüngste polnisch-jüdische Vergangenheit kritisch hinterfragt. Die fluide Verarbeitung unterschiedlichster Motive - jüdischer, romantisch-messianischer, popkultureller - steht nicht nur für einen enttabuisierten Umgang mit Narrativen über den Holocaust, die der Postmoderne quasi schutzlos ausgesetzt werden. Sondern auch den identitätsstiftenden Narrativen der polnischen Kultur, welche zum großen Teil in der Romantik fußen, werden kritische und dekonstruktive Gedanken entgegengesetzt. Mit der Besprechung dieser drei Fallbeispiele kann lediglich ein Einblick in die beschriebenen Tendenzen gewährt werden, welche, ohne Anspruch auf Allgemeingültigkeit, von den ausgewählten Texten bebildert werden sollen.

\section{Ausgewählte Fallbeispiele}

\section{Joanna Bators Familienroman Chmurdalia (2010)}

Joanna Bators Chmurdalia kann als eine Art Familienroman bezeichnet werden, der entlang der Lebensgeschichten der drei Frauen Dominika, Jadzia und Grażynka erzählt wird. In die Narrative dieser Figuren sind auch Erzählstränge eingeflochten, die polnisch-jüdische Geschichten und Identitäten zum Thema haben. Dominika Chmura, mit deren Autounfall die Erzählung im Jahr 1989 beginnt, erfährt erst nach dem Erwachen aus dem Koma von ihrem jüdischen Großvater Ignacy Goldbaum, der während seines Besuchs bei Oma Zofia Opfer eines antisemitischen Brandanschlages wird und stirbt. Ihre Mutter Jadzia Chmura, die ebenso tragisch und überraschend von ihrem leiblichen Vater erfährt, scheint seit Dominikas Unfall und dem Tod Zofias und Ignacys in einer Starre verhaftet zu sein, aus der sie sich gegen innere Ängste vor dem Anderen und antisemitischen Einstellungen nur schwer lösen und für ihre eigene jüdische Geschichte bzw. für ihren leiblichen Vater öffnen kann. Die dritte Protagonistin und Freundin der Chmuras, Grażynka Rozpuch, hat den Zweiten Weltkrieg und den Holocaust noch als Kind erlebt und dient im Roman als Schlüssel zur Erzählung der vergangenen polnisch-jüdischen Geschichte von Kamieńsk, einer kleinen Stadt zwischen Łódź und Częstochowa. 
Diese Erzählstränge bilden in der komplexen Struktur des Romans keine geschlossene Fläche, sondern sind verwoben mit zahlreichen und wechselnden anderen Strängen, wodurch der Roman je nach Blickwinkel andere Schwerpunkte setzt und , die jüdische Geschichte' nur eine von vielen ist. In Bezug auf die Konzeption des Textes heißt das, dass die Narrative jüdischer Thematik denselben narrativen Mitteln ausgesetzt werden, z. B. der Fiktionalisierung über die Grenze des Authentischen/Realen hinaus, wie die anderen fiktionalen Erzählungen und man von einem enttabuisierten literarischen Umgang sprechen kann. In Chmurdalia bedeutet das ein hierarchiebefreites Ineinander polnisch-jüdischer Narrative, welches eine kritische Auseinandersetzung mit Rollenbildern und Mega-Narrativen der polnischen Kultur transportiert.

Aus diesem Werk sollen nun zwei der Erzählstränge herausgegriffen werden, um das Ineinander, das wie eine konzeptionelle literarische Wanderung durch das Unbewusste konstruiert wird, zu veranschaulichen. Zum einen soll Dominikas ,hyphenisierte' Herkunft diskutiert werden, anhand derer im Roman jüdische Identität als etwas Verborgenes/Unbewusstes thematisiert wird und ein sensibles psychologisches Relief der späten Entdeckung der eigenen jüdischen Identität in Bezug auf die Ich-Konstruktion gezeigt wird. Zum anderen wird über Grażynka, die den Holocaust selbst noch als Kind erlebte und damit Zeitzeugin nicht nur der Shoah, sondern auch der längst verschwundenen Welt der polnisch-jüdischen Schtetl ist, ein fiktionaler - ja mystischer - Rückblick in diese Welt geworfen werden.

Die Auseinandersetzung mit der polnisch-jüdischen Geschichte des Zweiten Weltkriegs und der Shoah wird in Chmurdalia um das Städtchen Kamieńsk platziert und als fiktive Erzählung mit Grażynka als Knotenpunkt literarisiert. Die Erzählung über Kamieńsk beginnt ganz abrupt, in Grażynkas Erinnerungen eingebettet, mit der Beschreibung seiner Bewohnerinnen, die nach dem Krieg ohne Bedauern die Häuser der vertriebenen und ermordeten jüdischen Nachbarinnen und Nachbarn bezogen, so als hätte es nie ein gemeinsames Leben in Kamieńsk gegeben und als wäre es der natürliche Lauf der Dinge, dass der jüdische Besitz „, [...] demjenigen zufallen [würde], der als Erster die Pfote danach ausstreckte, denn so ist das Leben“ (Bator 2013: 81).

Ohne direkte emotionale Gewichtung wird in Chmurdalia die nicht vorhandene Identifikation mit den jüdischen Mitmenschen klar formuliert und damit nicht vor einer kritischen und unverblümten Darstellung des polnischen Antisemitismus Halt gemacht. Dem Verdrängen der Figuren im Text steht die Erzählinstanz gegenüber, die die gemeinsame polnisch-jüdische Geschichte in ihrer Ambivalenz erzählt, womit der Text einen differenzierten und analytischen Blick auf die verschwiegene gemeinsame Geschichte wirft. So finden wir neben der im polnischen Diskurs verbreiteten und lange dominierenden Retterfigur - wie sie im Roman z. B. von den Nonnen, die Grażynka ins Kloster retten, dargestellt wird - in Chmurdalia eine große Spannbreite an Figuren und Motiven, die sich nicht auf das unbunte 
Schwarz und Weiß reduzieren lassen. Interessant ist dabei vor allem die Figur des Kamieńsker Friseurs Tadeusz Kruk, welcher beim Abholen einer Perücke in eine Razzia gerät und daraufhin als ,Homosexueller' inhaftiert wird. Doch seine Geschichte reiht sich nicht in polnische Opfer-Narrative ein, denn Kruk überlebt das Konzentrationslager wohlgenährt dank der Gunst, beinahe Verbundenheit eines SS-Offiziers und seiner mit Lust zur Perfektion getriebenen Schur der weiblichen Häftlinge. Tadeusz Kruk wird in Chmurdalia nicht nur als pervers und skrupellos beschrieben, sondern regelrecht als Inkarnation des Bösen dargestellt, womit seine Figurenkonzeption sich von der Opfer-Darstellung polnischer Narrative über den Holocaust absetzt und einen bis heute von konservativen Kreisen als Tabu empfundenen Bruch mit der Reinheit und Unschuld des nationalen-martyrologischen Opfers vollzieht. In Bezug auf den polnisch-jüdischen Diskurs erfüllt Chmurdalia in der fiktiven Erzählung über Kamieńsk somit nicht nur die Funktion eines intellektuellen Zeugnisses, indem es den polnischen Holocaust-Diskurs um eine nuancierte und kritische Beschreibung polnischer Realitäten im Zusammenhang mit dem Zweiten Weltkrieg und der Shoah erweitert, sondern betont auch marginale Narrative der polnischen Literatur über den Holocaust. Neben Tadeusz Kruk rücken die Schicksale der im Konzentrationslager inhaftierten Frauen in den Vordergrund, auch derer, die in den Bordellen der Lager zur Prostitution gezwungen wurden. Thematisch kann hier nicht von einer neuen Enttabuisierung des polnischen Diskurses gesprochen werden, da in der Literatur bereits durch Schriftsteller wie Tadeusz Borowski oder Marian Pankowski Brüche mit tradierten Opfer-Narrativen vollzogen wurden. Tabuisiert wurden allerdings weitgehend Erotik, Sexualität und Prostitution in der Lagerthematik. Bei Bator wird der literarische Umgang mit dem Holocaust erst durch die literarische Aufarbeitung des Themas zu einer Neuheit und Gradwanderung auf der Tabugrenze. Die Sprache der Erzählung über Kamieńsk bedient sich realitätsentfremdender stilistischer Mittel und rückt die ganze verschwundene polnisch-jüdische Welt von Kamieńsk in ein fantastisches, fast mystisches Licht. Nicht nur Gegenstände haben übersinnliche Kräfte, so leuchtet z. B. der Nachttopf in der Speisekammer der Teetanten so hell, dass ",[...] man auch in der tiefsten Nacht [in der Speisekammer] kein Licht anzünden brauchte" (Bator 2013: 72) sondern auch der jüdische Fotograf Ludek hat eine magische Gabe - er sieht den nahenden unnatürlichen Tod der Fotografierten und damit die Shoah auf seinen Portraits voraus. Auch stilistische Figuren knüpfen an die Sprache von Märchen an. So ist der Text nicht nur reich an Allegorien und Vergleichen, sondern auch vereinfachend, die Straßennamen von Kamieńsk sind z. B. schlicht nach ihrem Verlauf benannt - "die Quere Straße", "die Kurze Straße" und "die Gerade Straße" (Bator 2013: 59) - oder die Bewohnerinnen und Bewohner mit einem sie charakterisierenden Adjektiv benannt, wie der melancholische Lehrer, oder die trikotierende Josephine. Auf diese Weise rückt Kamieńsk in seiner sprachlichen Aufarbeitung in märchenhafte Ferne, stößt sich fast provokant von seiner historischen Realität 
$\mathrm{ab}$, die von Bator schriftstellerisch äußerst versiert nicht nur fiktionalisiert, sondern mystifiziert wird.

In der Narration Dominikas geht es dagegen um eine Entmystifizierung der Vergangenheit - und zwar durch das Erfahren der Geschichte der eigenen Herkunft. Dieser Prozess wird in Chmurdalia von verschiedenen Traumata überschattet, welche im Text über den Geruch nach verbranntem Fleisch, den Dominika seit ihrem Unfall nicht aus der Nase bekommt, in Kongruenz gebracht werden. So entsteht eine Verbindung von Dominikas Unfall mit der antisemitisch motivierten Ermordung Oma Zofias und Ignacy Goldbaums sowie mit den vermutlich traumatischen Kriegserfahrungen Oma Zofias, welche ihr Leben lang viel Essig versprühen musste um den Geruch nach verbranntem Fleisch zu verdrängen. Im Roman wird der Geruch sogar direkt als Erbe beschrieben: „Die Enkelin hat es ganz eindeutig von ihrer Großmutter geerbt: Zofia Maślak, Jadzias Mutter, hat ihn ihr Leben lang gerochen [...]" (Bator 2013: 113). Interessanterweise dient Dominika im Roman jedoch nicht der Erzählung einer Spurensuche der Enkelgeneration, sondern vielmehr des an die Oberfläche tretenden Unterbewussten, so scheint es fast sinnbildlich, dass die Enkelin den Tod der Großmutter nicht bei Bewusstsein, sondern im Koma erlebt. Die fehlende Kommunikation der verdrängten Heterogenität der eigenen Geschichte und Herkunft, in Dominikas Fall bezieht sich das auf den verschwiegenen jüdischen Großvater und den ungekannten russischen Großvater väterlicherseits, machen die eigene Herkunft zu einer unbekannten, wenn auch unterbewussten Komponente und problematisch für die Identitätskonstruktionen der Enkelgeneration. In Bators Roman behilft sich die Protagonistin aus diesem Dilemma, indem sie sich auf eine Wanderschaft weg vom Ursprung der Traumata begibt. Die Wanderschaft weg von Polen ermöglicht ihr eine andere Perspektive auf den eigenen Ursprung, was als Motiv auch von Emigrant_innen polnisch-jüdischer Herkunft geäußert wird. So schreibt z. B. (die Wissenschaftlerin) Dorota Głowacka, die 1989 aus Polen nach Kanada emigrierte, über ihre jüdischen Wurzeln: „Nagle coś, do czego lepiej było się w Polsce ,nie przyznawać ${ }^{\prime}[. .$.$] , stało się powodem do dumy i przyczyną społecznej$ akceptacji“ (Głowacka 200). Ähnlich scheint die Wanderschaft in Bators Roman für Dominika, als Enkelin eines jüdischen Holocaust-Überlebenden, zu funktionieren. Sie hilft ihr nicht nur einen Umgang mit den Traumata des polnischen kollektiven Gedächtnisses zu finden, sondern birgt auch die Inspiration etwas Neues am Ort der Erinnerung aufzubauen:

[...] sie setzt sich an den Rand des Felsabhangs und schaut aufs Meer. Dann spürt sie, dass sich dieser Drang nicht überlisten lässt. Plötzlich steigt ihr der Gestank verbrannten Fleisches in die Nase, sie sieht die Asche des Hauses ihrer Großmutter Zofia in Zalesie vor sich, die leere, verkohlte Stelle, an der sie etwas Neues aufbauen muss (Bator 2013: 494). 
Wie ein solcher Versuch mutet auch Bators Text an, in dem Zusammenhänge und Resonanzen konstruiert werden, die ein diskursives Miteinander polnisch-jüdischer Erzählungen möglich machen. Die zeitliche und persönliche, da nicht biographische Distanz und die Stufe der Fiktionalisierung, die eingangs als enttabuisierte Form des Sprechens beschrieben wurde, ermöglichen eine Wieder-/Neuaneignung der polnisch-jüdischen Geschichte. Aus getrennten Erzählsträngen wird eine neue Geschichte geflochten, die tradierte Narrative von illusionärer Homogenität einerseits und fester Grenzen zwischen dem Eigenen und dem Fremden andererseits, in ihrer Fluidität auflöst. Jüdische Geschichten und Identitäten werden ohne gesonderte literarische Behandlung in einen polnischen Familienroman integriert, der sein breites Publikum eben nicht gesondert mit dem Etikett der ,Holocaust-Literatur' erreicht. Dennoch vollzieht Chmurdalia das der ,Holocaust-Literatur' immanente Zeugnisablegen und Gedenken, welches zwar in der Erinnerungsgemeinschaft aktualisiert und differenziert wird, jedoch auch durch die märchenhafte Stilisierung entfremdet wird. Diese Entfremdung kann als Grandwanderung interpretiert werden, die sich zwischen dem Versuch bewegt, die vom Holocaust vernichtete und vom Wandel der Zeit in eine fremde Ferne entrückte polnisch-jüdische Geschichte für die Gegenwart zugänglich zu machen, und dem Abrutschen in ein die historischen Ereignisse verharmlosendes Trugbild. Die Verfremdung des Holocaust zu einer märchenhafte Geschichte, die in ihrer Abstraktion vom konkreten historischen Ereignis abgelöst zur Fabel verkommt, kann auch kritisch als Manöver interpretiert werden, dass sich die Geschichte leichter bekömmlich aneignet und damit schmerzhafteren Reflektionen entzieht. Ob es nun an der Zeit ist, sich vom Schmerz zu lösen und Neues an seiner Stelle zu bauen, und ob dies in Chmurdalia gelingt, kann an dieser Stelle leider nicht zu Ende diskutiert werden - ein interessanter und mutiger erster Schritt ist es allemal.

\section{Politik und Erotik in Jarosław Kamińskis Rozwiązła (2011)}

Der polnische Journalist, Erzähler und Dramatiker Jarosław Kamiński legte mit seinem 543 Seiten zählenden Buch ein interessantes Romandebüt vor, das von der polnischen Literaturkritik fast einstimmig hoch gelobt wurde ${ }^{4}$. Kamińskis Buch nimmt in der zeitgenössischen Literatur Polens einen besonderen Platz ein, vor allem was die vom Autor aufgegriffenen Tabuthemen: Xenophobie, Antisemitismus, Kollaboration, Feminismus, Homosexualität und Prostitution betrifft. Für Aufsehen sorgte aber auch sein außergewöhnliches Erzähltalent, sein langanhaltender „epischer Atem“. Kamiński gehört zu jenen Vertreter_innen der zeitgenössischen polnischen Literatur, denen es mit Erfolg gelang den Teufelskreis des ewig Polnischen zu durchbrechen und eine selbstverliebte polnische Nabelschau zu ver-

4 In Deutschland ist der Autor bisher im Zusammenhang mit dem gemeinsam mit Marc Metzger geschriebenen Drehbuch zum Film Nachmieter (2011) bekannt geworden. 
meiden; geradezu lustvoll bricht er dabei mit herkömmlichen Tabus. Körperliches, Erotisch-Sexuelles wie auch Grenzüberschreitungen unterschiedlicher Art werden in Kamińskis Roman in einer (für die polnische Literatur) ungewohnten Freizügigkeit dargestellt. Doch den Text allein auf diese Themen zu reduzieren, wäre oberflächlich und nicht gerechtfertigt. Ganz im Gegenteil, Rozwiazła kann durchaus zur anspruchsvollen polnischen Literatur gezählt werden, auch wenn Sex and Crime zu wichtigen, unverzichtbaren Komponenten des Buches gehören. Der Romantitel, Rozwiazła, bezieht sich auf die attraktive Hauptprotagonistin des Romans, Zofia Rogala, und scheint zunächst den einseitigen Bezug auf das Erotisch-Sexuelle zu bestätigen. Doch der Titel ist mehrdeutig. Im positiven Sinne könnte man ihn mit "Libertine" wiedergeben. Rozwiazła bedeutet aber auch „die Lasterhafte, Zügellose, Ausschweifende, Sittenlose", mit einem eindeutig negativen Bezug. Auf jeden Fall verweist der Titel auf die Sonder- und Außenseiterstellung der Protagonistin. Er bezieht sich aber auch auf einen angeblich gleichnamigen Ort im ehemaligen Ostpreußen, der den deutschen Namen „Lasterdorf“ trug. Auch eine Figur, die im Verlauf des Romans für Zofia Rogala immer mehr an Bedeutung gewinnt, trägt den Familiennamen „Lasterdorf". Kamińskis Roman kann unterschiedlich gelesen und interpretiert werden, als Lebensgeschichte der "freizügigen“, geheimnisvollen Zofia Rogala mit ihrem komplizierten, „,anderen“, ungewöhnlichen Leben. Verantwortlich dafür sind private, persönliche Umstände, aber auch die vom Autor gut recherchierten und behutsam reflektierten historischen, gesellschaftspolitischen und kulturgeschichtlichen polnischen Themen im gesamteuropäischen Kontext, die sich hinter der Lebenserfahrung und Tragik dieser wie auch der anderen Romanfiguren verbergen. Über die persönlichen Schicksale der Figuren fließt polnische und europäische Geschichte in die Handlung ein; der historische, gesellschaftliche, politische wie auch kulturhistorische Hintergrund rückt im Erzählverlauf immer mehr in den Vordergrund und kann als eine weitere interessante Lesart angesehen werden. Im Mittelpunkt des Romans steht zunächst die Beziehung einer gebildeten, gestandenen, selbstbewussten Frau zu ihrem siebzehn Jahre jüngeren Liebhaber, einem Studenten (Adam Czerski) der Warschauer Akademie der Schönen Künste. Zunächst halten beide ihre ungewöhnlich erscheinende Beziehung geheim, doch letztendlich soll Adam Zofia endlich seiner Familie vorstellen, die in den Masuren, eben in jenem Ort mit dem sprechenden Namen „Rozwiązła“ (Lasterdorf) lebt. Doch die Familienbegegnung wird zu einem Desaster, nicht allein wegen der "unschicklichen“ Beziehung des Sohnes zu einer wesentlich älteren Frau, die ihn außerdem noch vor der Homosexualität "gerettet" haben soll. Hinter dem eindeutig ablehnenden Verhalten vor allem von Adams Mutter und dessen Großvater Edward, steckt allerdings mehr als nur reine Abneigung. Erst im Verlauf der Erzählung offenbaren sich immer deutlicher zahlreiche Geheimnisse und Rätsel, die die Figuren miteinander verbinden, die erst zum Schluss, wie in einem Krimi, aufgeklärt werden. Das bisherige Leben Zofias, ihre ungeklärte Herkunft (sie wuchs in einem Waisenhaus 
auf), ist wohl das größte Geheimnis. Zu den sexuellen, erotischen und feministischen Aspekten gesellt sich im Roman ein weiterer Aspekt, den man detektivistischkriminalistisch nennen könnte, der zu einer erhöhten Spannung im Erzählverlauf beiträgt. Die Geheimnisse haben ihren Ursprung in der Zeit des Zweiten Weltkriegs und des Holocaust, als jüdische Kinder mit Hilfe falscher "arischer" Papiere und Namen gerettet wurde und $\mathrm{u}$. a. in Klöstern versteckt wurden. Der zeitliche Bogen des Romans wird vom Zweiten Weltkrieg über das weltweite Exil, das zeitliche Umfeld des Jahres 1968 bis in die unmittelbare Gegenwart Polens gespannt. Als einer der ersten Schriftsteller_innen macht Kamiński die sogenannten Märzereignisse von 1968 mit den Studentenunruhen und Machtkämpfen innerhalb der Polnischen Vereinigten Arbeiterpartei, den ",antizionistischen“ Kampagnen und Verfolgung zum Thema seines Buches. Dabei ist das verhängnisvolle Jahr 1968 auch mit den perfiden Aktionen des polnischen Geheimdienstes verbunden, die sich vor allem gegen Intellektuelle, Künstler_innen und Jüd_innen richteten.

Zofia sucht als angebliches Waisenkind nach ihren Eltern. Letztendlich erfährt sie über den Großvater von Adam, wer ihr Vater war; sie findet ihre eigene jüdische Identität als Chaja Goldfarb heraus und erleidet eine schwere Identitätskrise.

\section{Igor Ostachowicz Noc żywych Żydów (2012) - mehr als eine Schauergeschichte}

Eine besondere Stellung in der zeitgenössischen polnischen Literatur nimmt der bisher noch nicht ins Deutsche übersetzte Roman Noc żywych Żydów von Igor Ostachowicz ein. Er kann zu den spektakulärsten Werken der fiktionalen Holocaustliteratur der Enkelgenration gezählt werden, die sich mit der nach 1945 entstandenen homogenen, monoethnischen und monokulturellen Realität Volkspolens besonders kritisch auseinandersetzt. In diesen Narrationen treten die wenigen noch in Polen verbliebenen Jüdinnen und Juden häufig in Gestalt von Phantomen auf; der polnische Antisemitismus existiert weiterhin, auch ohne die reale Präsenz von Jüdinnen und Juden (Trepte 2014: 39-56). Man kann Ostachowiczs Buch auch als eine eigenwillige Form der Trauerarbeit, als ein „verspätetes Kaddisch“ ansehen (Staszczyszyn). Die in Polen verbreitete Haltung der eigenen (National)Geschichte versucht oft das Jüdische zu marginalisieren, um dafür die eigenen Heldentaten wie auch die eigenen Toten, nicht zuletzt im Sinne des polnischen Messianismus, besonders herauszustellen. Gegen dieses historische Gepäck, den polnischen Buckel im Gombrowicz'schen Sinne, das keine differenzierten, objektiven Lesarten ermöglicht, da die Pol_innen weitgehend mit dem Beklagen der eigenen Opfer beschäftigt sind, wendet sich der Schriftsteller ausdrücklich (Wolff-Powęska). Aus der Perspektive des nach dem schwierigen Transformationsprozess entstandenen neuen Polens setzt sich Ostachowicz in seinem Buch mit der Vernichtung, Vertreibung, der Erinnerung und Bewältigung des Holocaust, mit den im wahrsten Sinne des Wortes in den Kellern verbliebenen Leichen auseinander. Das dem Erdboden gleichgemachte 
Warschau, vor allem das Warschauer Ghetto, ja ganz Polen wird vom Erzähler als ein einziger riesiger Friedhof angesehen, der von den Überlebenden augenblicklich ausgeplündert wird:

Geboren wurde ich und ich wohne bis heute in einer Stadt der Goldgräber. Sie kamen aus der niedergebrannten Ebene hierher. Nach goldenen Zähnen und silbernen Löffeln Suchenden. Es zogen sie der Rauch der Ruinen und der Brandgeruch der Leichname reicher Bürger an [...]. Warschau ist keine schöne Stadt, doch man liebt hier das Leben in seiner reinsten Form (Ostachowicz 5, Übersetzung H.C.T.).

Und dieses neue, von Konsum und Kommerz bestimmte Leben schließt das Andenken an die Toten weitgehend aus, die einst die Straßen des Warschauer Ghettos bevölkerten. Ungewollt bringen sie sich letztendlich selbst in Erinnerung. Aus den Kellern der zerstörten Häuser, auf dessen Fundamenten gleich nach dem Ende des Zweiten Weltkriegs eilig neue sozialistische Häuserblocks errichtet wurden, steigen plötzlich die Untoten, die ermordeten Jüdinnen und Juden, Zombies gleich, in die Oberwelt auf. Es kommt zu direkten, bizarr-grotesken Begegnungen, zu eigenwilligen Konfrontationen der Lebenden mit den Untoten. Ostachowicz nimmt in seinem Buch ein zentrales Motiv der ,jüdischen Keller" und der jüdischen Geister, die auch als Zeug_innen wie in Andrzej Barts Fabryka muchołapek (2008) auftreten können, auf und baut es weiter aus. Verwiesen sei in diesem Kontext des Weiteren auf Sylwia Chutniks Debütwerk Kieszonkowy atlas kobiet (2008), in dem das alte Kellerverlies mit dem Geist der ermordeten (jüdischen) Mutter eine zentrale Rolle spielt:

Der Keller im Mietshaus in der Opaczewska-Straße. Er konnte von den Menschen nicht mehr normal genutzt werden, trotz Generalüberholung und sorgfältigstem Abkratzen der Leichen von den Wänden. Die Kellermauern hatten Dinge gesehen, wonach sie kein Fahrrad, keinen Liegestuhl und kein Eingewecktes für den Winter mehr beherbergen konnten. Solche Orte sind Gedenkstätten. Was aber soll man tun, wenn eine Gedenkstätte Teil der Gegenwart ist: ein Haus, das von Lebenden bewohnt wird? Die Keller zuschütten, so tun, als wäre hier nie etwas passiert. Einen kleinen Laden dort unten eröffnen, einen Club im Wohngebiet. Ein Solarium. Den Ort des Verbrechens mit neuen, positiven Assoziationen belegen. Ihn den Leuten zurückgeben. Habt keine Angst, kommt her und sonnt euch. Der Krieg ist längst vorbei, eigentlich hat es ihn nie gegeben (Chutnik 2012: 100).

Der Keller wird bei Chutnik, ebenso wie auch bei Ostachowicz, zu einem Symbol der verdrängten jüdischen Geschichte und des Holocaust. Niemand hatte die Untoten gerufen, nein, sie waren von ganz allein gekommen und geisterten nun durch die polnischen Städte: denn "die Wahrheit kommt immer ans Licht [...]" 
(Chutnik 2012: 107). Und so kam die jiddische Mamma immer häufiger aus dem Keller herauf zu ihrer Tochter Maria (sic!):

Sie legte die Hände auf Marias Stirn, starrte in ihre Pupillen. Einmal hob sie ihr Kopftuch etwas an und zeigte die Wunde von dem Gewehr, an der sie gestorben war. In dem Schorf konnte man Marias Schicksal erkennen: Ein gleichmäßiger Strich, ein Rinnsal, das zum Mund floss. Eine schmale Linie, die in dem Loch zwischen den Zähnen endete. Eine Begegnung wie aus den Ahnenfeiern, Zauberei, Simsalabim im Kellermoder (Chutnik 2012: 107).

Mit großem Erfolg geht Sylwia Chutnik in ihrem in Polen und Israel aufgeführten Bühnenstück Muranooo (2012) erneut auf die besondere Topographie und Geschichte des Warschauer Stadtbezirks Muranów ein, in dem sich das Ghetto befand und jüdische Geister bis heute spuken ${ }^{5}$. Mit Chutnik vergleichbar verbindet auch Ostachowicz die unerwartete, plötzliche Präsenz der jüdischen Geister bzw. Untoten mit dem modernen Leben der heutigen polnischen Bewohner_innen des Stadtviertels. Dabei vermischen sich bei beiden Schriftsteller_innen Elemente der schwarzen Komödie, des Grotesken mit Elementen des Horrors und Absurden.

Ostachowiczs Roman Noc żywych Żydów ist in einer bewusst schockieren und provozieren wollenden Weise geschrieben. Der Erzähler hinterfragt nicht nur die jüngste jüdisch-polnische Vergangenheit, sondern richtet sich ganz bewusst gegen gängige Ikonen der polnischen Holocaustliteratur; er thematisiert den Holocaust als Horror-Fiction im Stil der Popkultur, in der modernen, z. T. vulgären Umgangs- bzw. Gossensprache der heutigen polnischen Jugend. Zielgerichtet werden Elemente der Komödie und des Horrors miteinander verknüpft. Man könnte Igor Ostachowiczs Roman auch als eine Fortführung, „eine Art Persiflage“ auf die bereits genannten Werke von Sylwia Chutnik, aber auch von Andrzej Barts Fabryka muchołapek lesen, hinter dessen "skurrilen Handlung sich der ewige Kampf zwischen Gut und Böse, Himmel und Hölle verbirgt" (Golebiowski 31). Ostachowiczs Text repräsentiert einen neuen, unverkrampften Umgang mit dem Holocaust, nicht oberflächlich und seicht, sondern mit entsprechendem Tiefgang, mit erstaunlicher Reife. Der (Anti)Held des Buches ist ein in der Transformationszeit in Polen sozialisierter Everyman, ein Fliesenleger von Beruf, allerdings mit einem höheren Bildungsgrad, der viel lieber als Handwerker arbeitet, weil er damit mehr Geld verdient, als würde er sein Leben als „Intellektueller" fristen. Mit seiner magersüchtigen und arbeitslosen Partnerin Chuda, der Mageren, lebt er in einem Wohnblock auf dem Gebiet des ehemaligen Ghettos. Da tauchen eines Tages plötzlich aus den Kellern des Blocks zahlreiche jüdische Geister, Zombies gleich, auf. Das ungleiche polnische Paar freundet sich mit der Jüdin Rachel (Rejczel) an, deren traumatisierte Seele

5 Vgl. dazu: http://culture.pl/pl/wydarzenie/chutnik-o-duchach-z-muranowa [Zugriff: 18.05.2016]. 
keine Ruhe finden kann und erst dann erlöst wird, wenn es ihr gelingt, ihr Trauma, nicht zuletzt durch das wiedergewonnene Lächeln, zu überwinden. Erst dann kann sie die Erde verlassen und in den Himmel kommen. Ein für polnische Leser_innen offenkundiger Bezug auf Adam Mickiewiczs Drama Dziady, in dem in einer heidnischen Szene die Geister der Verstorbenen beschworen werden:

So auch die Seelen von zwei kleinen Kindern, die nicht ohne Hilfe der Lebenden ins Himmelreich gelangen können: ,Hört und wollet wohl erwägen dies Gebot von Gottes Hand: Wer nicht Bitternis gekannt, hat im Jenseits keinen Segen.' Erst als sie je eine ,Prise Bitterrinde' bekommen, können sie die Erde verlassen und gen Himmel aufsteigen (Przyborowska 1).

Auch bei Ostachowicz helfen die Lebenden den Untoten Eingang ins Paradies zu finden. Um sie zum erlösenden Lächeln zu bringen, begeben sie sich in ein irdisch-profanes Shopping-Center, in ein Konsum-Paradies. Mit Rachel und anderen jüdischen Zombies ziehen sie in das supermoderne "Arkadia" (sic!) ein, um sich dort einem hemmungslosen Konsumrausch hinzugeben. Ihnen folgen immer mehr jüdische Seelen, die sich in diesem neuen Arkadien mit einem ihnen bisher völlig unbekannt und fremd gebliebenen Lebensstil vertraut machen, den die Lebenden für sie finanzieren sollen. Alsbald ist die ganze Warschauer Innenstadt mit den jüdischen Untoten aus Muranów bevölkert. Auf sie eröffnen polnische Neonazis plötzlich eine gewalttätige Hetzjagd, ein Verweis auf das Problem des alten wie neuen polnischen Antisemitismus. Dabei kommt es zu einer folgenschweren Wandlung eines vom Teufel besessenen Neonazis, von „Jemand Böses“ (Ktoś Zły) zu „Jemand Vollkommen Böses“" (Ktoś Zupełnie Zły), der zu einer absoluten Bedrohung für alle (untoten) jüdischen Seelen und ihre irdischen Freund_innen wird. Dem cleveren polnischen Fliesenleger gelingt es jedoch mit einer List „seine“ Juden und Jüdinnen zu retten ${ }^{6}$, allerdings verliert er dabei sein eigenes Leben und wird vom Engel Uriel zum Jüngsten Gericht abgeholt.

Zwei sich eigentlich ausschließende Matrix-Formen durchdringen in Ostachowiczs Buch einander auf provokante und grotesk-komische Weise. Die verschwiegene, tabuisierte Wahrheit über ein besonders finsteres Kapitel polnisch-jüdischer Vergangenheit kommt auf eine ungewollte und furchteinflößende Art und Weise ans Tageslicht. Das plötzliche Auftreten der (jüdischen) Leichen aus den Kellern, zweifelsohne Verkörperung all dessen, was nicht (mehr) sichtbar sein sollte, führt nunmehr als Spuk und Horror zu einer diametralen Umkehrung der bisher geltenden Ordnung und Werte, es kommt zu einer karnevalesk-grotesken Begegnung mit dem weitgehend verdrängten Schicksal der einstigen jüdischen Nachbar_innen.

6 Auch hier ist ein Bezug zu einem Film von Agnieszka Holland gegeben: W ciemności - In Darkness (2011). 
Igor Ostachowicz geht es, wie auch anderen Schriftsteller_innen seiner Generation, nicht zuletzt um das amputierte polnische Gedächtnis - gemeinsam wollen sie sich auf Spurensuche begeben, sich bewusst erinnern. Ob der Roman Ostachowiczs auch als eine Art "moderner, eigensinniger Bildungsroman" (Sokalska), wohl hauptsächlich auf den Fliesenleger bezogen, verstanden werden kann, sei dahingestellt, auf jeden Fall ist das Buch ein gewagter Versuch, sich mit der Thematik des Holocaust auf eine völlig neue Weise auseinanderzusetzen. Dabei weist der Roman in seinen unterschiedlichen Narrationen ein verblüffendes Geflecht an unterschiedlichen Motiven, Anspielungen, literarischen wie filmischen Assoziationen auf.

Auffallend ist bei der dritten und vierten Generation in Bezug auf die Thematisierung des Holocaust gerade die insgesamt deutlich zunehmende Visualisierung. Mit dem iconic bzw. visual turn kommt es zu einem deutlichen Paradigmenwechsel (Dalle Vacche). Das zeigt sich bereits im Titel des Romans, eine Paraphrase auf den Film Night oft he Living Dead (1968) (Die Nacht der lebenden Toten) von George A. Romero. Der Titel Ostachowiczs spielt im polnischen Kontext des Weiteren auf ein Buch der namhaften polnischen Literaturwissenschaftlerin Maria Janion an: Do Europy tak, ale razem z naszymi umartymi (2000).

Vorgefertigte Bilder (Filme, Computerspiele, Internet) spielen in den Köpfen der polnischen Protagonist_innen eine wichtige Rolle, selbst die Erzählstrategien scheinen am ehesten denen eines Computerspiels zu entsprechen. Die Präsenz von Versatzstücken (Filme, Computerspiele, TV-Serien, Literatur) ist fast überall gegeben. Dabei arbeiten die „meisten dieser Filme mit einer im Bewusstsein der Zuschauer tief verankerten Holocaust-Ikonographie: ausgehungerte Häftlinge, SS-Männer, die Stacheldrahtzäune um das Lager, die rauchenden Schornsteine, der Judenstern, das Ankunftstor in Auschwitz mit seiner Arbeit-macht-frei-Inschrift, u. a. m." (Sruk 199). Doch Ostachowicz verzichtet in seinem Text zumeist auf derartige Ikonen.

Der Roman endet mit einer dramatischen, gewalttätigen, grotesk gestalteten Szene, mit einem apokalyptischen Finale, in dem der Protagonist in der wahren Hölle, in Auschwitz, die größte Maschinerie des absoluten Bösen kennenlernen muss, wo Menschen Menschen in Öfen verbrennen.

Wie in den drei Beispielen darzustellen erhofft wurde, geht es in der zeitgenössischen polnischen Literatur, die sich mit dem Thema des Holocaust beschäftigt, um eine Gradwanderung entlang der bisher abgesteckten moralischen Grenzen der ,Holocaust-Literatur', die sich, wie überblicksartig dargestellt, jedoch nicht erst nach 1989 thematisch und auch interdisziplinär zu verschieben und gewissermaßen zu weiten begannen. In den gewählten Beispielen der jüngeren Generation werden vergessene, aber zentrale Puzzleteile der polnischen Geschichte, darunter eben auch jene, die jüdisches Leben zum Thema haben, aus den dunklen Ecken des Vergessenen und Verdrängten hervorgeholt und im grellen Licht gegenwärtiger, unbefangener Untersuchungen, mit all ihren Formen und Strategien, verifiziert. Dadurch wird auf der visuellen Ebene sicher eine gewisse Nivellierung des Holo- 
caust vorangetrieben, wie sie hier im Text mit Alvin Rosenfeld bereits angesprochen wurde. Allerdings zeigen alle drei angeführten Beispiele, dass diese Nivellierung in ihrer Bedeutung vor allem positiv zu bewerten ist, da sie mit einer allgemeinen und zugleich gesamtgesellschaftlichen Steigerung von Relevanz und Wahrnehmung der Holocaustthematik einhergeht. Mit den künstlerischen Mitteln, die der zeitgenössischen Literatur zur Verfügung stehen, deren Einschätzung jedoch eine Frage des Geschmacks bleiben sollte, gelingt es in diesen fiktiven Texten nicht-jüdischer Autor_innen, die Erinnerung, die Diskussion und die Wahrnehmung der polnischjüdischen Geschichte zu sich wechselseitig ergänzenden, zumeist ,passenden' und für alle sichtbaren Puzzleteilen eines gemeinsamen, nicht nur polnische Geschichte betreffenden Gesamtbilds zu machen.

\section{Überraschende Debüts}

Die polnisch-jüdische Schriftstellerin Irena Wiszniewska kommt in Ihrem Beitrag „,Illegal im Reich der Geister'. Jüdisches Leben im heutigen Polen“ (Wiszniewska 2016a) zur Erkenntnis, dass „Polen - wegen des Holocausts und des Mangels an Empathie oder gar der Feindseligkeit der Nachbarn - ein ,verfluchtes Land“" (Wiszniewska 2016a: 95) sei. Dieser Befund bezieht sich sowohl auf die Holocaustüberlebenden im Ausland als auch in Polen, auf Vertreter_innen der zweiten und dritten Generation, die auf der Suche nach ihrer Familiengeschichte nach ihren „verschütteten Wurzeln" (Wiszniewska 2016a: 97) sind. Piotr Paziński, Schriftsteller und Chefredakteur der jüdischen Monatszeitschrift Midrasz, äußert sich dazu wie folgt:

Juden, die in Polen geblieben sind, sind hier quasi entgegen der herrschenden Weltordnung geblieben, entgegen der Geschichte, die von der Katastrophe gezeichnet ist. Sie sind ein Zeichen dieser Katastrophe, eine Narbe, eine Spur. Im Grunde genommen sind sie Illegale, denn sie leben unrechtmäßig in diesem Reich der Geister (zitiert nach: Wiszniewska 2016a: 98).

Viele vermeintlich ,polnische' Autor_innen hatten ihr ,jüdisches Coming-out erst ziemlich spät, zum Einen, weil sie von ihrer jüdischen Herkunft erst sehr spät erfuhren, zum Anderen, weil sie nach dem Ende des Zweiten Weltkriegs zunächst bei ihren Tarn- bzw. veränderten Familiennamen blieben wie z. B. Bożena Umińska, die erst im Nachvollzug zu ihrem jüdischen Namen Keff zurückfand. Hinzu kommt aber auch die bewusste Verdrängung des Erlebten in der Vergangenheit (Ubertowska 2007), die Todesangst, sowie Verfolgungen und Demütigungen. Von besonderem Interesse sind auch Debüts, die versuchen aus dem bisher geschlossenen Orbis interior des Fremd-bzw Andersseins, der Exklusion und der Selbstmarginalisierung auszubrechen. Dazu gehört $u$. a. der aufschlussreiche Fall des polnischen Literatur- 
wissenschaftlers Michał Głowiński. Bei Głowiński kommt es zu einem verspäteten doppelten Coming-out, als Jude und als Homosexueller. Dabei stellt die Problematik von Holocaust und (Homo)Sexualität eine besondere Narration innerhalb der polnischen Gegenwartsliteratur dar, auf die hier jedoch nicht eingegangen werden kann (Ubertowska 2014b: 291-311). Im polnisch-jüdischen Kontext kommen des Weiteren auch Fragestellungen des Feminismus und Gender (Bąk-Zawalski 157-169) hinzu, die, etwa Mitte der siebziger Jahre des 20. Jahrhunderts beginnend, zu den besonders polarisierenden und kontrovers diskutierten Bereichen der Holocaustforschung gehören (Ubertowska 2014c: 113-134, 2014a: 135-159).

Der Feminismus geht manchmal mit einer doktrinären und kämpferischen Haltung einher. [...] Ich möchte einfach das, was man über jüdische Frauen nicht weiß, ergänzen. Das interessiert mich sehr und ich finde es wichtig, weil man ihnen nur selten eine Stimme gegeben und zugehört hat und ihre Texte nur aus der männlichen Perspektive kommentiert wurden (Wiszniewska 2016b: 118).

Die späte Selbstwahrnehmung der eigentlichen, authentischen Herkunft führt zu einem andersartigen Blick auf das eigene Leben, das Jüdische und den Holocaust (Gosk, Karwowska). Ähnlich wie die Vertreter_innen der zweiten Generation betrachten die Repräsentant_innen der dritten und vierten Generation ihr jüdisches Erbe völlig anders als die der sogenannten Erlebnisgeneration. Allerdings leidet auch die zweite Generation oft noch unter den vererbten, weitergegebenen und wiedererlebten Traumata, so u. a. während der antisemitischen Kampagne in Polen von 1968 und der von ihr ausgelösten Emigrationswelle in den Westen: „Ich hatte das Gefühl, den Holocaust zu erleben. Manche meinen, mein Vergleich sei völlig übertrieben, aber so habe ich damals empfunden" (Wiszniewska 2016a: 110). Dabei kommt es im Zusammenhang mit den nicht verarbeiteten Traumata zu einer eigenwilligen „Prolongierung des Holocaust” (Czapliński 2012: 299). Vertreter_innen der zweiten Generation bezeichnen sich in ihrer Funktion nicht selten als eine Art "Scharnier" zwischen den Holocaustüberlebenden resp. Holocaustzeug_innen und den Nachfolgenden, während die Vertreter_innen der Enkelgeneration sich häufig als eine Art Mittler_innen und Brückenbauer_innen sehen. In diesem Zusammenhang stellt sich die Frage nach der Literatur als einem Therapeutikum, welches hauptsächlich in Bezug auf den Holocaust "traumatische Erinnerungen" (Caruth) durch eine "heilende Erinnerungskultur" bzw. eine gezielte "Literaturtherapie“ (Helbig-Mischewski 29-38) wie auch Vertreibung und Heimatverlust zu lindern, wenn nicht sogar auszukurieren vermag (Muschg). Weitgehend frei von solchen Vorstellungen ist das Denken, die Gefühlslage, die Perspektive und Einstellung bei den Vertreter_innen der dritten und vierten Generation, der "sudden generation of Jews" (Reszke 92f.). Auch wenn sie immer noch mit dem Schatten der jüdischen und polnischen Vergangenheit leben müssen und die "Schlacht um die Erinnerung", 
die „nationale Psychoanalyse“ (Wiszniewska 2016a: 102) in Polen weiter fortgesetzt wird und unbequeme, schmerzliche, bisher verdrängte bzw. tabuisierte Themen (wie u. a. der Antisemitismus) aufgegriffen werden (müssen).

Festzuhalten gilt, dass erst nach dem demokratischen Umbruch von 1989/1990, "als die Angst vorbei war", ein neues ,jüdisches Leben“ wieder "aufblühen“ konnte (Wiszniewska 2016b: 116). Die wiederentdeckte bzw. wieder angeeignete jüdische Identität mit Generationenkonflikten und einer spezifischen Gedächtnistradierung wird als eine interessante Alternative, aber auch als ein Bedürfnis der Selbst-Authentifizierung (wieder)entdeckt. Es ist eine Alternative, die Sicherheit und Unhinterfragbarkeit zu versprechen scheint. So spricht die 1978 in Wrocław geborene Schriftstellerin, Regisseurin, Kultur- und Literaturwissenschaftlerin Katka Reszke in ihrer Studie Return of The Jew. Identity Narratives of The Third Post-Holocaust Generation of Jews in Poland (2013) von einer Wiederkehr des Jüdischen in Identitätsnarrationen der dritten Post-Holocaustgeneration nach dem demokratischen Umbruch in Polen und verweist darauf, nach welchen Mustern jüdische Identität rekonstruiert werden kann. „Weil es Polen ist, wo die jüdische Kultur so vollständig herausgerissen wurde, ist hier die Entdeckung selbst einer kleinsten jüdischen Wurzel was ganz Besonderes, anders als woanders in der Welt" (Luba). Gleichzeitig wächst das authentische Interesse für die ausgelöschte jüdische Welt, für den Judaismus und die jiddische Sprache bei vom Jüdischen und dem Holocaust gänzlich unbetroffenen Autor_innen. Diese wollen, obwohl sie mit,dem Jüdischen' bisher eigentlich nichts zu tun hatten, nunmehr zielgerichtet über einen Teil der jüdischen Kultur schreiben, der in Polen für immer vernichtet ist, was von besagten Autor_innen als großer Verlust angesehen wird. Das zeigt sich $u$. a. in der Gestalt eines Philosemitismus mit z. T. exotisch wie geheimnisvoll anmutenden jüdischen Themen, die in unterschiedlicher Gestalt in polnische Narrationen vom zeitgenössischen Roman, über den Comic bis hin zum Krimi aufgenommen werden. Auf diese Weise etablieren sich neuartige Narrationen mit anderen Schreibstrategien, mit provokanten Darstellungsformen, vor allem bei den gegen Ende der 1970er, Anfang der 1980er Jahre geborenen literarischen Vertreter_innen der dritten Generation. Verwiesen sei an dieser Stelle auch auf die engen Beziehungen von Postmoderne und Holocaust (Eaglestone).

Die „stärkere Rückbesinnung“ der letzten Jahre führt aber auch zu einer erneuten Hinwendung zu frühen literarischen Zeugnissen des Holocaust, die „neu publiziert und kontextualisiert werden“ (Roth 2015: 16). Dazu gehören auch Narrative des Rewritings (Young) bzw. der (Neu)Interpretationen von Ikonen der jüdischpolnischen Literatur wie z. B. Bruno Schulz (Biller). Außerdem werden die früheren Zeugnisse auch in der gegenwärtigen literatur- und geschichtswissenschaftlichen Forschung neu fokussiert ${ }^{7}$. Olga Tokarczuk meint, dass die neue Aufarbeitung wie

7 Vgl.: Holý, Jiř́, hg. The Aspects of Genres in the Holocaust Literatures. Praha: Akropolis, 2015; Krupa, Bartłomiej Opowiedzieć Zagładę. Polska proza i historiografia wobec holokaustu (1987 - 2003). Kraków: 
auch die „Verklärung“ der jüdischen Identität vielen Polen „eine Art starke, verschworene Gemeinschaft" verspricht, "an der es der heutigen polnischen Gesellschaft so fehlt" (Wodecka).

Zusammenfassend kann bei den Vertreter_innen der Kinder- und Enkelgeneration beobachtet werden, dass sie ,das Jüdische' ebenso wie den Holocaust literarisch thematisieren und auf eine völlig andersartige Weise eine verschüttete, sich nicht mehr nur auf ,das Polnische' beschränkende Identität im Sinne einer demokratischen, weit gefassten und offenen Polonität ergründen und wiederentdecken möchten. Damit wollen sie zu einer notwendigen Klärung eines sie immer wieder aufs Neue bewegenden Tatbestands beitragen, der in der Fragestellung gipfelt, weshalb die mehr als neunhundert Jahre zählende Geschichte von Pol_innen und Jüd_innen nur eine Geschichte des Nebeneinanders, bestenfalls eines Miteinanders geblieben ist. Nicht zuletzt aus diesem Grunde stellen sie sich die Aufgabe, in dieses Bergwerk der Erinnerung einzufahren, es zu ergründen, um all seine Tiefen aufs Neue zu erforschen. Ihr Ziel besteht dabei darin, die auch weiterhin schmerzlich spürbaren Lücken im kollektiven Gedächtnis, nicht zuletzt unter Berücksichtigung der selbstzerstörerischen Folgen, zu schließen: Denn es geht ihnen heute nicht mehr allein nur um die Vergangenheit, um Tod und erlittenes Leid, sondern um die Gegenwart und Zukunft von Menschen, unabhängig von ihrer ethnisch-kulturellen Herkunft.

\section{BIBLIOGRAPHIE}

Assmann Aleida, Frevert Ute. Geschichtsvergessenheit - Geschichtsversessenheit. Vom Umgang mit deutschen Vergangenheiten nach 1945. Stuttgart: DVA, 1999.

Bajohr Frank, Löw Andrea. Der Holocaust. Ergebnisse und neue Fragen der Forschung. Frankfurt am Main: Fischer, 2013.

Bąk-Zawalski, Aleksandra. „The Reception of Holocaust Literature with Feminist Context in Poland Based on the Example of Bożena Umińska-Keff and Ruth Klüger`s Works“. The Aspects of Genres in the Holocaust Literatures in Central Europe. Hg. J. Holý. Praha: Akropolis, 2015. S. 157-170.

Bart, Andrzej. Fabryka muchołapek. Warszawa: W.A.B., 2008.

Bator, Joanna. Chmurdalia. Warszawa: W.A.B., 2010.

Bator, Joanna. Wolkenfern. Berlin: Suhrkamp, 2013.

Biller, Maxim. Im Kopf von Bruno Schulz. Köln: Kiepenhauer und Witsch, 2013.

Błoński, Jan. „Biedni Polacy patrzą na getto“. Tygodnik Powszechny 41(2) (1987). S. 1-4.

Breysach, Barbara. Schauplatz und Gedächtnisraum Polen. Die Vernichtung der Juden in der deutschen und polnischen Literatur. Göttingen: Wallstein, 2005.

Universitas, 2013; Breysach, Barbara Schauplatz und Gedächtnisraum Polen. Die Vernichtung der Juden in der deutschen und polnischen Literatur. Göttingen: Wallstein, 2005. 
Caruth, Cathy. Trauma: Explorations in Memory. Baltimore: Johns Hopkins University Press,1995.

Chutnik, Sylwia. Kieszonkowy atlas kobiet. Kraków: Korporacja Ha!art, 2008.

Chutnik, Sylwia. Weibskram. Berlin: Vliegen Verlag, 2012.

Chutnik, Sylwia. Muranooo. Uraufführung, 2012.

Clendinnen, Inga. Reading the Holocaust. New York: Cambridge University Press, 2002.

Czapliński, Przemysław. „Zagłada - niedokończona narracja polskiej nowoczesności“. Ślady obecności. Hg. S. Buryła, A. Molisak. Kraków: Universitas, 2010. S. 337-392.

Czapliński, Przemysław. „Holocaust und Profanierung“. Der Holocaust in der polnischen Erinnerungskultur. Hg. A. Wolff-Powęska, P. Forecki. Frankfurt am Main, 2012. S. 299-313.

Dalle Vacche, Angela. The Visual Turn: Classical Film Theory and Art History. New Brunswick: Rutgers University Press, 2003.

Dörner, Andreas. Politainment. Politik in der medialen Erlebnisgesellschaft. Frankfurt am Main: Suhrkamp, 2001.

Doschek Jolanta, Simonek, Stefan, hg. Slawische Popkultur. Wien: PAN, 2015.

Eaglestone, Robert. The Holocaust and the Postmodern. Oxford: Oxford University Press, 2008.

Engelking, Leszek. „Laleczki na sprzedaż. Zabawa w Holokaust i handel holokaustem“. Holokaust Šoa - Zagłada v české, slovenské a polskéliteratuře. Hg. J. Holý. Praha: Univerzita Karlova, 2007. S. 79-95.

Finkelstein, Norman. Die Holocaustindustrie. Wie das Leiden der Juden ausgebeutet wird. München: Piper, 2000.

Głowacka, Dorota. „Quo Vadis? Ojczyzna, tożsamość wyobrażona i »mój malutki los«”. PL - Tożamość wyobrażona. Hg. J. Tokarska-Bakir. Warszawa: Czarna Owca, 2013. S. 198-215.

Głowiński, Michał. „Wprowadzenie“. Stosowność i forma. Jak opowiadać o Zagładzie? Hg. M. Głowiński. Kraków: Universitas, 2005. S. 7-20.

Głowiński, Michał. Kręgi obcości. Opowieść autobiograficzna. Kraków: Wydawnictwo Literackie, 2010.

Golebiowski, Anja. „Die Geister der Vergangenheit. Trauma und Psychoanalyse in der polnischen Gegenwartskunst und -literatur". The Holocaust in the Central European Literatures and Cultures since 1989 / Der Holocaust in den mitteleuropäischen Literaturen und Kulturen nach 1989. Hg. R. Ibler. Stuttgart: Ibidem, 2014. S. 17-38.

Gosk Hanna, Karwowska Bożena. (Nie)obecność. Pominięcia i przemilczenia w naracjach XX wieku. Warszawa: Elipsa, 2008.

Gross, Jan Tomasz. Sąsiedzi: Historia zagłady żydowskiego miasteczko. Sejny: Fundacja Pogranicza, 2000.

Gross, Jan Tomasz. Upiorna dekada. Trzy eseje o streotypach na temat Żydów, Polaków, Niemców i komunistów 1939-1948. Kraków: Universitas, 2001.

Hartmann, Geoffrey. Holocaust Remembrance: The Shapes of Memory. Oxford: Blackwell, 1994.

Helbig-Mischewski, Brygida. „Time to say fuck you - o matce, ojczyźnie i okrucieństwie ofiar“. Pogranicza 4 (2009). S. 29-38.

Hobermann, James Lewis. „The Past Can Hold a Horrible Power“ The New York Times. 2013. Web. 27.06.2016, <http://www.nytimes.com/2013/10/27/movies/aftermath-a-thriller-directed-by-wladyslaw-pasikowski.html?_r=0>.

Holý Jiří, hg. Holokaust - Šoa - Zagłada v české, slovenské a polské literatuře. Praha: Univerzita Karlova, 2007. 
Holý Jiří, hg. The Aspects of Genres in the Holocaust Literatures. Praha: Akropolis, 2015.

Hüchtker, Dietlind. „Männlichkeit im Sozialismus und Pop in Polen. Ein Fundstück“. Dekonstruieren und doch erzählen. Polnische und andere Geschichten. Hg. J. Heyde et al. Göttingen: Wallstein, 2015. S. 66-72.

Janion, Maria. Do Europy tak, ale razem z naszymi umartymi. Warszawa: Sic!, 2000.

Kamiński, Jarosław. Rozwiązła. Warszawa: W.A.B., 2011.

Kowalska-Leder, Justyna. „Literatura polska ostatniego dziesięciolecia wobec Zagłady - próby odpowiedzi na nowe wyzwania“. Zagłada Żydów. Studia i materiały 10 (2014). S. 768-802.

Krupa, Bartłomiej. „Die Intensivierung der Holocaust-Diskussion“. Der Holocaust in der polnischen Erinnerungskultur. Hg. A. Wolff-Powęska, P. Forecki. Frankfurt am Main et al.: Peter Lang, 2012. S. 195-213.

Krupa, Batłomiej. Opowiedzieć Zagładę. Polska proza i historiografia wobec holokaustu (1987 - 2003). Kraków: Universitas, 2013.

Krupa, Bartłomiej. „Polish Holocaust Discourse 1945-2013“. The Aspects of Genres in the Holocaust Literatures in Central Europe. Hg. J. Holý. Praha: Akropolis, 2015. S. 25-40.

Littell, Jonathan. Les bienveillantes. Paris: Èditions Gallimard, 2006.

Luba, Arkadiusz. „Wie polnische Jugendliche mit ihren jüdischen Wurzeln umgehen“. Deutschlandradio Kultur. 2015. Web. 18.05.2016. <http://www.deutschlandradiokultur.de/wiedergefundenes-judentum-wie-polnische-jugendliche-mit.1079.de.html?dram:article_id=331522>.

Miłoszewski, Zygmunt. Ziarno prawdy. Warszawa: W.A.B., 2015.

Miłoszewski, Zygmunt. Uwikłanie. Warszawa: W.A.B., 2014.

Miłoszewski, Zygmunt. Gniew. Warszawa: W.A.B., 2014.

Muschg, Adolf. Literatur als Therapie? Ein Exkurs über das Heilsame und das Unheilbare. Frankfurter Vorlesungen. Frankfurt am Main: Suhrkamp, 1981.

Ostachowicz, Igor. Noc żywych Żydów. Warszawa: W.A.B., 2012.

Pakier, Małgorzata. „Postmemory‘ jako figura reflekcyjna w popularnym dyskursie o Zagładzie“. Kwartalnik Historii Żydów 2 (2005). S. 195-208.

Pankowski, Marian. „Nie ma Żydówki“. Twórczość 63(8) (2007). S. 5-21.

Probst, Lothar. „Founding Myths in Europe and the Role of the Holocaust". New German Critique 90 (2003). S. 45-48.

Przyborowska, Maria. „World War Z auf Polnisch“. 2013. eb. 27.06.2016. <http://www.novinki.de/ przyborowska-maria-world-war-z-auf-polnisch> 27.06.2016.

Reichel, Peter. Erfundene Erinnerung. Weltkrieg und Judenmord in Film und Theater. München: Carl Hanser Verlag, 2001.

Reszke, Katja. Return of the Jew. Identity Narratives of the Third Post-Holocaust Generation of Jews in Poland. Boston: Academic Studies Press, 2013.

Rosenfeld, Alvin H. „Popular Culture and the Politics of Memory“. The End of Holocaust. Hg. A. Rosenfeld. Bloomington: Indiana University Press, 2013. S. 14-32.

Rottenberg, Anda. Proszę bardzo. Warszawa: W.A.B., 2009.

Roth, Markus. „Gattung Holocaustliteratur? Überlegungen zum Begriff und zur Geschichte der Holocaustliteratur". The Aspects of Genres in the Holocaust Literatures in Central Europe. Hg. J. Holý. Praha: Akropolis, 2015. S. 13-23. 
Szczygielski, Marcin. Arka czasu. Warszawa: Wydawnictwo Piotra Marciszuka STENTOR, 2013.

Sokalska, Arlena. „«Noc żywych Żydów» Ostachowicza: Zamiast ,szokującego horroru’ niezła postmodernistyczna powieść“. Polska online (2012). Web. 20.05.2016. <http://www.polskatimes.pl/ artykul/552459,noc-zywych-zydow-ostachowicza-zamiast-szokujacego-horroru-niezla-postmodernistyczna-powiesc, 2,id,t,sa.html >.

Sruk, Marija. „Vom ausgrenzenden zum verbindenden Lachen: Die dritte Generation und der Holocaust in Am Ende kommen Touristen (2007) und Hannas Reise (2013)“. The Aspects of Genres in the Holocaust Literatures in Central Europe. Hg. J. Holý. Praha: Akropolis, 2015. S. 197-212.

Staszczyszyn, Bartosz. „Noc żywych Żydów - Igor Ostachowicz“. 2012. Web. 27.06.2016. <http://culture.pl/pl/dzielo/noc-zywych-zydow-igor-ostachowicz>.

Szczęsna, Joanna. „Holocaust i kicz“. Gazeta Wyborcza (2011). Web. 21.06.2016. <http://wyborcza. pl/1,76842,9086918,Holocaust_i_kicz.html>.

Trepte, Hans-Christian. „Kinder und Enkel des Holocaust erzählen. Neue Perspektiven in der Holocaustliteratur". The Holocaust in the Central European Literatures and Cultures since 1989. Hg. R. Ibler. Stuttgart: Ibidem, 2014. S. 39-56.

Trepte, Hans-Christian. „Einige Bemerkungen zur Problematik Holocaust und Comic im polnischen Kontext". The Aspects of Genres in the Holocaust Literatures in Central Europe. Hg. J. Holý. Praha: Akropolis, 2015. S.143-155.

Ubertowska, Aleksandra. Świadectwo, trauma, głos. Literackie reprezentacje Holokaustu. Kraków: Universitas, 2007.

Ubertowska, Aleksandra. Holokaust. Auto(tanato)grafie. Warszawa: PAN, 2014.

Ubertowska, Aleksandra. „Gender i Holokaust: strategie przetrwania, zasady tekstotwórcze“. Holokaust. Auto(tanato)grafie. Hg. A. Ubertowska. Warszawa: PAN, 2014a. S. 135-159.

Ubertowska, Aleksandra. „Kręgi obcości, podwójne wyjście? Projekt autobiograficzny Michała Głowińskiego“. Holokaust. Auto(tanato)grafie. Hg. A. Ubertowska. Warszawa: PAN, 2014b. S. 291-311.

Ubertowska, Aleksandra. „Niewidoczność, sprawczość, podmiot. Perspektywa feministyczna i genderowa w badaniach nad Holokaustem“. Holokaust. Auto(tanato)grafie. Hg. A. Ubertowska. Warszawa: PAN 2014c. S. 113-134.

Wiszniewska, Irena. „«Illegal im Reich der Geister». Jüdisches Leben im heutigen Polen“. Jahrbuch Polen 2016. Minderheiten. Deutsches Polen-Institut Darmstadt. 2016a. S. 95-106.

Wiszniewska, Irena im Gespräch mit Bella Schwarcman-Czarnota „Wiegenlieder in Jiddisch“. Jahrbuch Polen 2016. Minderheiten. Deutschen Polen-Institut Darmstadt. 2016b. S. 107-124.

Wodecka, Dorota. „Das ist kein Land für Ketzer, ein Gespräch mit Olga Tokarczuk“. Gazeta Wyborcza (2015), zitiert nach Wiszniewska, Irena (2016a). S. 105.

Wolff-Powęska, Anna. „Historia z recyklingu“. Gazeta Wyborcza (2013). Web. 23.06.2016. <http://wyborcza.pl/magazyn/1,124059,14919321,Historia_z_recyklingu.html>.

Young, James E. Writing and Rewriting the Holocaust: Narrative and the Consequences of Interpretation. Bloomington: Indiana University Press, 1988. 